\title{
Helmintofauna de Paroaria dominicana (Linnaeus, 1758) oriundos do tráfico de animais silvestres
}

\author{
Helminth fauna of Paroaria dominicana (Linnaeus, 1758) from illegal wildlife trade \\ Helmintofauna de la Paroaria dominicana (Linnaeus, 1758) procedente del tráfico de animales \\ salvajes
}

Rayana Emanuelle Rocha Teixeira ORCID: https://orcid.org/0000-0001-5287-2853 Universidade Federal da Bahia, Brasil E-mail: rayy.emanuelle@ hotmail.com

Marilene Souza Oliveira

ORCID: https://orcid.org/0000-0002-1585-8902 Universidade Federal da Bahia, Brasil E-mail: souzaoliveira.mari@gmail.com

Isabela Sousa Prado

ORCID: https://orcid.org/0000-0003-2994-8949 Universidade Federal da Bahia, Brasil

E-mail: isabela.prado@ufba.br

Edma Santos de Antonio

ORCID: https://orcid.org/0000-0003-1280-8472 Universidade Federal da Bahia, Brasil

E-mail: edma.santos@ufba.br

Antônio Iderval Sodré Neto

ORCID: https://orcid.org/0000-0003-0136-7210 Universidade Federal da Bahia, Brasil

E-mail: neto.sodre@gmail.com

Matheus Santos dos Anjos

ORCID: https://orcid.org/0000-0001-6120-8698 Universidade Federal da Bahia, Brasil

E-mail: matheussantosdosanjos6@gmail.com

Laize Tomazi

ORCID: https://orcid.org/0000-0003-0977-8508 Universidade Federal da Bahia, Brasil E-mail: laizetomazi@yahoo.com.br

Patrícia Belini Nishiyama

ORCID: https://orcid.org/0000-0002-9495-6171 Universidade Federal da Bahia, Brasil E-mail: profpatriciabelini@gmail.com

Márcio Borba da Silva

ORCID: https://orcid.org/0000-0002-6497-7924 Universidade Federal da Bahia, Brasil E-mail: biolmarcioborba@gmail.com

Ricardo Evangelista Fraga

ORCID: https://orcid.org/0000-0001-9345-4869 Universidade Federal da Bahia, Brasil E-mail: ricardoefraga@ hotmail.com

\section{Resumo}

O tráfico de animais silvestres é um problema com repercussões ecológicas e sociais. Além de causar o declínio de diversas populações naturais, esta prática tem consequências diretas sobre os indivíduos capturados, como quadros de imunossupressão que podem resultar na manifestação de variados patógenos. No Brasil, o tráfico de aves silvestres se destaca como uma das modalidades mais populares desta prática. As espécies mais procuradas usualmente possuem 
atrativos estéticos ou sonoros, como muitos exemplares Passeriformes. O cardeal-do-nordeste (Paroaria dominicana) é uma destas espécies, chegando a ser considerada uma das mais traficadas do país. Neste trabalho, analisamos 26 exemplares de Paroaria dominicana provenientes de uma ação de resgate executada pelo IBAMA. Foram realizadas técnicas de necropsia e sedimentação espontânea para verificar a presença de helmintos e ovos nos órgãos dos exemplares analisados, bem como pesagem e mensuração dos cadáveres. Encontramos a presença de espécies de nematódeos em 05 exemplares; todos pertencentes ao gênero Diplotriaena, incluindo a espécie Diplotriaena delirae, registrada pela primeira vez na helmintofauna de Paroaria. dominicana. Observamos a presença de ovos característicos do gênero Diplotriaena e Heterakis, este último também inédito a helmintofauna de Paroaria dominicana até o presente trabalho. A presença de novas espécies na helmintofauna de Paroaria dominicana pode estar associada ao contato direto com outras espécies silvestres durante a captura e o transporte ilegal, bem como as condições sanitárias associadas a essas etapas do tráfico de aves.

Palavras-chave: Helmintofauna; Paroaria dominicana; Tráfico de aves.

\begin{abstract}
Illegal wildlife trade is a problem with ecological and social repercussions. In addition to causing the decline of several natural populations, this practice has direct consequences over the captured individuals, such as immunosuppression conditions that can result in the manifestation of various pathogens. In Brazil, wild bird trafficking stands out as one of the most popular forms of this practice. The most sought-after species usually have aesthetic or sound appeals, like many Passerine species. The Northeastern Cardinal (Paroaria dominicana) is one of these species, considered one of the most trafficked in the country. In this work, we analyzed 26 specimens of Paroaria dominicana from a rescue action carried out by IBAMA. Necropsy and spontaneous sedimentation techniques were performed to check the presence of helminths and eggs in the organs of the specimens analyzed, as well as weighing and measuring of the cadavers. We found the presence of nematode species in 05 specimens; all belonging to the genus Diplotriaena, including the species Diplotriaena delirae, registered for the first time in the Paroaria dominicana helminth fauna. We observed the presence of eggs characteristic of the genus Diplotriaena and Heterakis, the latter also unprecedented in the helminth fauna of Paroaria dominicana until the present work. The presence of new species in the helminth fauna of Paroaria dominicana may be associated with direct contact with other wild species during capture and illegal transport, as well as the sanitary conditions associated with these stages of bird trafficking.
\end{abstract}

Keywords: Helminth fauna; Paroaria dominicana; Illegal bird trade.

\title{
Resumen
}

Tráfico de animales salvajes es un problema con repercusiones ecológicas y sociales. Además de provocar el declive de varias poblaciones naturales, esta práctica tiene consecuencias directas sobre los individuos capturados, como inmunosupresión que puede resultar en la manifestación de varios patógenos. En Brasil, el tráfico de aves silvestres se destaca como una de las formas más populares de esta práctica. Las especies más buscadas suelen tener atractivos estéticos o sonoros, como muchos Passeriformes. El cardeal-do-nordeste (Paroaria dominicana) es una de estas especies, llegando a ser considerado uno de los más traficados del país. En este trabajo analizamos 26 ejemplares de Paroaria dominicana de una acción de rescate realizada por IBAMA. Se realizaron técnicas de necropsia y sedimentación espontánea para verificar la presencia de helmintos y huevos en los órganos de los especímenes analizados, así como pesar y medir los cadáveres. Encontramos la presencia de especies de nematodos en 05 especímenes; todos pertenecientes al género Diplotriaena, incluida la especie Diplotriaena delirae, registrada por primera vez en la fauna de helmintos de Paroaria. dominicana. Observamos la presencia de huevos característicos del género Diplotriaena y Heterakis, este último también inédito en la fauna de helmintos de Paroaria dominicana hasta el presente trabajo. La presencia de nuevas especies en la fauna de helmintos de Paroaria dominicana puede estar asociada al contacto directo con otras especies silvestres durante la captura y transporte ilegal, así como las condiciones sanitarias asociadas a estas etapas del tráfico de aves.

Palabras clave: Helmintofauna; Paroaria dominicana; Tráfico de aves.

\section{Introdução}

O Brasil é um dos principais fornecedores de animais para o tráfico internacional de animais silvestres, movimentando ilegalmente uma quantia estimada em aproximadamente R $\$ 2.5$ bilhões por ano (Renctas, 2001). As consequências ecológicas relacionadas a esta prática são severas, tornando-a uma das principais causas do declínio populacional de diversas espécies silvestres (Regueira \& Bernard, 2012). Fato que se deve, principalmente, às más condutas de captura e às condições precárias de transporte e venda (Alves et al., 2012). Anualmente, no Brasil, são retirados cerca de 38 milhões de animais silvestres de seus ambientes naturais; destes, estima-se que 4 milhões chegam a ser vendidos vivos (Leape \& Brito, 2013).

Os altos níveis de estresse aos quais os animais traficados são expostos, causam consequências negativas ao sistema 
imunológico dos mesmos. Comumente, estes indivíduos apresentam quadros de imunossupressão, podendo resultar na manifestação de doenças, desenvolvimento de infecções e associação de parasitas (Sanches, 2008). O principal fator associado à transmissão de patógenos entre espécies silvestres é o contato direto entre os indivíduos durante as etapas de transporte e venda (Karesh et al., 2005). A translocação de espécies silvestres entre diferentes regiões expõe os parasitas associados a novos potenciais hospedeiros, tornando os padrões de patogenicidade imprevisíveis e possibilitando a transmissão de patógenos, muitas vezes causadores de zoonoses, entre diferentes espécies (Aguirre, 2010).

A média anual de animais recebidos pelos Centros de Triagem de Animais Silvestres (CETAS) no Brasil, é maior que 38 mil espécimes, sendo que mais de 80\% são aves, com a maioria proveniente do tráfico (Destro et al., 2012). Os motivadores por trás do comércio ilegal de aves são variados. Uma das preferências dos compradores são as espécies canoras ou de atração estética. Desta forma, a ordem dos Passeriformes é uma das mais populares no tráfico de aves silvestres (Costa et al., 2018). O “cardeal-do-nordeste" Paroaria dominicana (Linnaeus, 1758) é uma das espécies de passeriforme mais traficada do país, fator responsável pela introdução de populações desta espécie a diversas regiões além de seu alcance natural (Rosa et al., 2019).

Neste trabalho analisamos a helmintofauna de 26 exemplares de $P$. dominicana apreendidos do tráfico de animais silvestres. Uma análise parasitológica sobre cadáveres de indivíduos que passaram pelo tráfico pode oferecer uma melhor compreensão acerca da transmissão de parasitas e zoonoses associadas, bem como um melhor entendimento da parasitologia nativa de $P$. dominicana.

\section{Metodologia}

Foram necropsiados 26 exemplares de $P$. dominicana, no período entre agosto e outubro de 2019. Os animais originam-se de um resgate realizado pelo Instituto Brasileiro do Meio Ambiente e dos Recursos Naturais Renováveis (IBAMA). Os indivíduos resgatados foram fornecidos para o presente estudo pelo CETAS de Vitória da Conquista-BA (CETAS-VCA) e vieram a óbito nas imediações deste CETAS antes de passarem por qualquer procedimento. As aves foram levadas ao Laboratório de Zoologia do Instituto Multidisciplinar em Saúde, Campus Anísio Teixeira, da Universidade Federal da Bahia, onde foram acondicionadas a $-20^{\circ} \mathrm{C}$, até o momento da necropsia. O presente projeto de pesquisa foi aprovado pela Comissão de Ética em Uso de Animais (CEUA-IMS/CAT- UFBA), Protocolo no 074/2019. Este trabalho trata-se de uma pesquisa quantitativa descritiva (Dalfovo et al., 2008; Pereira et al., 2018).

Previamente à necropsia, foi realizada a pesagem individual de todos os animais, utilizando balança analítica digital. Depois de pesados, deu-se início às etapas da necropsia: (i) os exemplares foram colocados em decúbito dorsal, (ii) foi realizado um corte longitudinal partindo da base do bico até a cloaca, (iii) os órgãos internos (pulmões, fígado, intestino, coração e cavidade abdominal) foram removidos e (iv) os órgãos retirados passaram imediatamente por avaliação.

Os parasitas encontrados nos órgãos foram submetidos a lavagem em água destilada e armazenados com identificação de acordo com o órgão e o exemplar de origem. Os parasitas foram identificados por meio das medidas e da caracterização do tridente, da espícula e dos ovos. As medidas foram realizadas com o auxílio de microscópio óptico com lente micrométrica e registros fotográficos foram realizados com microscópio trinocular. A identificação seguiu os padrões morfológicos propostos por Vicente et al. (1983). Os ovos dos parasitas foram identificados por sedimentação espontânea do líquido do intestino com o auxílio de microscópio óptico.

As medidas morfométricas (peso e tamanho) dos exemplares parasitados foram comparadas com as dos exemplares não-parasitados por meio de um teste $\mathrm{T}$ de Welch para comparação de médias. A taxa de prevalência foi calculada dividindo o número de aves parasitadas por determinada espécie de parasita pelo número total de aves examinadas; a abundância média foi calculada pelo número de parasitas encontrados de determinada espécie pelo número total de aves examinadas; e a intensidade média de infecção foi calculada pelo número de parasitas encontrados de uma espécie em particular dividido pelo número total 
de aves parasitadas com essa espécie em particular (Bush et al., 1997).

\section{Resultados}

A necropsia dos 26 exemplares de $P$. dominicana encontrou parasitas presentes em 5 indivíduos (0.19). A partir da análise dos órgãos dos infectados, 17 helmintos foram encontrados, destes 11 foram identificados (todos do gênero Diplotriaena) (Tabela 1) e 6 não puderam ser identificados devido a estruturas corporais comprometidas. Além dos helmintos Diplotriaena inteiros, em uma das 5 aves parasitadas, foram encontrados ovos expostos do gênero Heterakis (sem a presença do helminto inteiro desse gênero). Na Figura 1, são mostrados os dois tipos de ovos encontrados no presente trabalho: (i) os ovos do gênero Diplotriaena, observados no interior do corpo dos helmintos; e (ii) os ovos de helmintos do gênero Heterakis, encontrados expostos, fora de qualquer corpo de helminto, descobertos pela técnica de sedimentação espontânea.

Tabela 1. Biometria dos parasitas identificados no presente trabalho $(\mathrm{n}=11)$.

\begin{tabular}{lccc}
\hline Espécie & Tridente & Ovos & Local de infecção \\
\hline Diplotriaena delirae & $0,24 \mathrm{~mm}$ & $0,030 \times 0,040$ & Cavidade abdominal \\
Diplotriaena delirae & $0,39 \mathrm{~mm}$ & $0,029 \times 0,042$ & Cavidade abdominal \\
Diplotriaena bargusínica & $0,10 \mathrm{~mm}$ & $0,031 \times 0,046$ & Cavidade abdominal \\
Diplotriaena bargusínica & $0,105 \mathrm{~mm}$ & $0,031 \times 0,046$ & Cavidade abdominal \\
Diplotriaena bargusínica & $0,11 \mathrm{~mm}$ & $0,029 \times 0,048$ & Cavidade abdominal \\
Diplotriaena henry & $0,11 \mathrm{~mm}$ & - & Intestino \\
Diplotriaena henry & $0,105 \mathrm{~mm}$ & $0,028 \times 0,045$ & Intestino \\
Diplotriaena henry & $0,095 \mathrm{~mm}$ & $0,030 \times 0,045$ & Intestino \\
Diplotriaena henry & $0,085 \mathrm{~mm}$ & $0,030 \times 0,048$ & Cavidade abdominal \\
Diplotriaena henry & $0,085 \mathrm{~mm}$ & $0,031 \times 0,042$ & Cavidade abdominal \\
Diplotriaena sp & $0,095 \mathrm{~mm}$ & $0,029 \times 0,045$ & Cavidade abdominal \\
\hline
\end{tabular}

Fonte: Dados da pesquisa.

A Tabela 1 mostra a biometria dos 11 helmintos identificados, todos do gênero Diplotriaena. 10 dos helmintos foram identificados a nível de espécie: 05 representantes da espécie Diplotriaena henry (Blanc, 1919), 03 da espécie Diplotriaena bargusinica (Skrjabin, 1917) e 02 da espécie Diplotriaena delirae (Pinto \& Noronha, 1970). Para 01 único helminto só foi possível a identificação até o nível de gênero: Diplotriaena sp. (Vicente, 1983). O tamanho dos tridentes variou entre 0,085 e $0,39 \mathrm{~mm}$. O tamanho dos ovos variou entre 0,028 e $0,031 \mathrm{~mm}$ para a dimensão menor; e entre 0,040 e $0,048 \mathrm{~mm}$ para a dimensão maior. A maioria dos parasitas $(n=8)$ foi encontrada na cavidade abdominal e os demais parasitavam o intestino $(\mathrm{n}=3)$. Os 2 espécimes de $D$. delirae foram encontrados em uma única ave. Os 3 espécimes de D. bargusínica foram retirados em duas aves. Os 5 espécimes de D. henry foram recuperados de quatro aves. O espécime de Diplotriaena sp. foi observado em uma única ave. 
Figura 1. Ovo característico do gênero Diplotriaena (A). Ovo característico do gênero Heterakis (B). Escala: $50 \mu$ m.

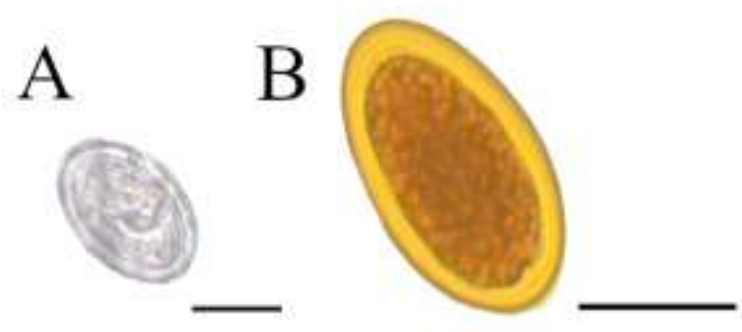

Fonte: Dados da pesquisa.

A Figura 1A mostra o ovo pertencente ao gênero Diplotraiena, eles são caracterizados por possuírem casca lisa e serem larvados. A Figura 1B mostra o ovo encontrado pertencente ao gênero Heterakis (Schrank, 1790), são de formato ovóide sem segmentação do conteúdo.

Das análises estatísticas obteve-se (i) a comparação de peso e tamanho corporal entre os indivíduos parasitados e não parasitados (Figura 2) e (ii) os valores de prevalência, intensidade média e abundância média dos parasitas nas amostras (Tabela 2).

Figura 2. Comparação de peso (A) e tamanho (B), entre os indivíduos parasitados e os não parasitados.
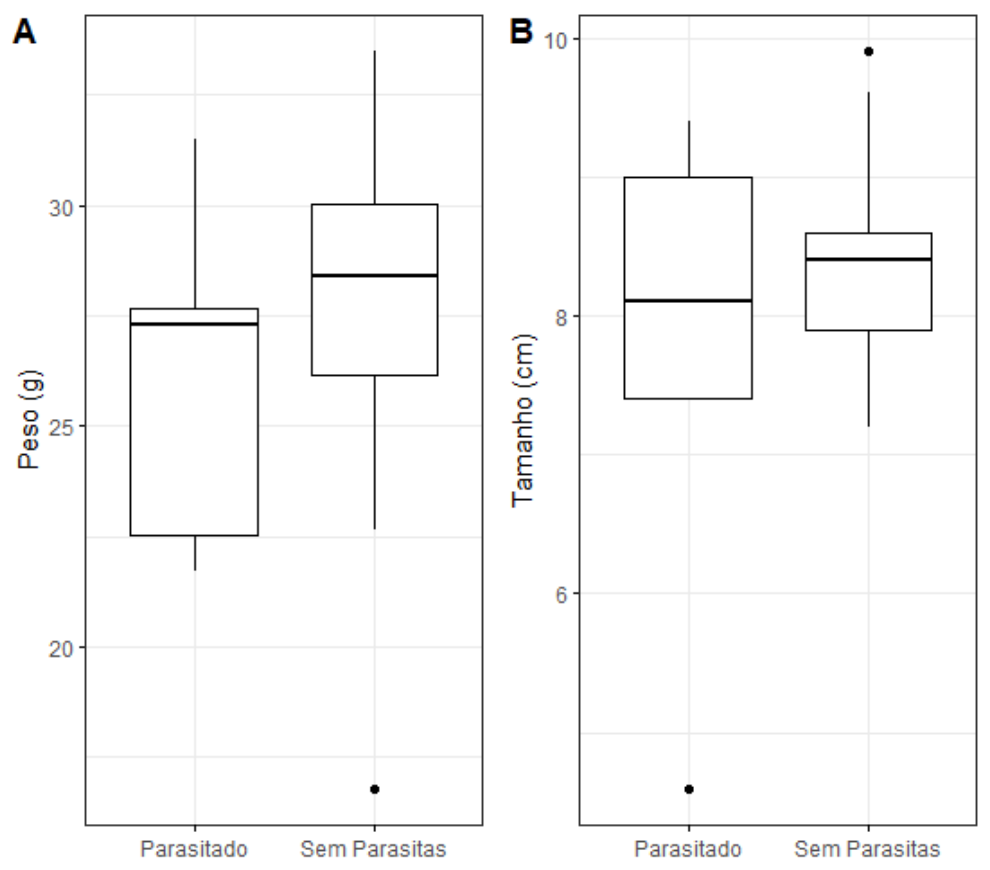

Fonte: Dados da pesquisa.

A Figura 2 mostra a comparação do tamanho e do peso do corpo entre as aves parasitadas e as não parasitadas. Não foram encontradas diferenças significativas entre o peso $(\mathrm{p}=0.4698)$ e o tamanho $(\mathrm{p}=0.4894)$, quando comparados os indivíduos parasitados com os não parasitados. 
Tabela 2. Prevalência, abundância média e intensidade média de parasitas em aves da espécie $P$. dominicana oriundas do tráfico de animais silvestres.

\begin{tabular}{lccc}
\hline Espécie & Prevalência & Abundância média & Intensidade média \\
\hline Diplotriaena delirae & 0.038 & 0.07 & 2 \\
Diplotriaena bargusínica & 0.076 & 0.11 & 1.5 \\
Diplotriaena henry & 0.153 & 0.19 & 1.25 \\
Diplotriaena sp. & 0.038 & 0.03 & 1 \\
\hline
\end{tabular}

Fonte: Dados da pesquisa.

A Tabela 2 traz o resultado das análises estatísticas, mostrando que (i) a espécie com maior prevalência e abundância média foi $D$. henry, (ii) a espécie com maior intensidade média foi $D$. delirae e (iii) a espécie com menor prevalência, abundância média e intensidade média foi Diplotriaena sp.. A prevalência dos helmintos identificados variou entre 0.038 e 0.153 ; abundância média variou entre 0.03 e 0.19 ; e a intensidade média variou entre 1 e 2 .

\section{Discussão}

Os nematódeos do gênero Diplotriaena foram prevalentes dentre os indivíduos parasitados. A especificidade deste gênero é restrita à infecção de aves, com registros de parasitismo em diversas ordens de aves silvestres (Vicente et al., 1983). Os hospedeiros intermediários deste gênero são, em sua maioria, artrópodes (Anderson, 2000). Sendo assim, a origem da infecção dos exemplares de $P$. dominicana analisados, pode estar relacionada ao fenômeno de insetivoria ocasional descrita na dieta da espécie (Silveira, 2012).

Esse é o primeiro registro da espécie $D$. delirae em exemplares de $P$. dominicana, bem como o primeiro registro desta espécie de helminto no nordeste do Brasil (Morais et al., 2018). Outro registro inédito foi a presença de ovos de indivíduos do gênero Heterakis em uma das aves parasitadas. Ambas as espécies de parasitas são características de outras espécies de aves comuns ao tráfico de animais silvestres (Boll et al., 2017; Morais et al., 2018). A transmissão de parasitas e possíveis zoonoses entre diferentes espécies é um fenômeno documentado no tráfico de aves silvestres (Karesh et al., 2005) e é associada a imunossupressão causada pelo estresse a qual são expostos na captura e no transporte, momentos onde os mesmos têm contato direto com muitos outros animais capturados (Aguirre, 2010; Sanches, 2008; Thompson et al., 2010). O registro de parasitas característicos de outras espécies na helmintofauna de $P$. dominicana no presente trabalho, pode estar associado a esse fator.

A presença de espécies do gênero Diplotriaena na helmintofauna de $P$. dominicana já foi descrita anteriormente na literatura, tanto para indivíduos de cativeiro quanto indivíduos de vida livre (Carvalho et al., 2006; Carvalho et al., 2007; Mascarenhas, 2008; Vicente et al., 1995). Nossos resultados contrastam com o estudo de Carvalho et al. (2006), que realizaram necropsia de 102 espécimes de $P$. dominicana e encontraram uma diversidade maior de parasitas, incluindo nematódeos, trematódeos e acantocéfalos; enquanto nossa análise identificou somente espécies de nematódeos.

Os exemplares parasitados não apresentaram diferenças significativas associadas ao peso e ao tamanho, quando comparados aos exemplares não-parasitados. Embora estudos parasitológicos anteriores relatem uma maior taxa de mortalidade quando em infecção em aves provenientes do tráfico (Sanches, 2008), a identificação de sinais clínicos associados à infecção parasitológica em aves silvestres é de difícil percepção, sendo muitas vezes inexistente ou apresentada de forma inespecífica (Tomás, 2014).

Em geral, os valores de prevalência, abundância média e intensidade média obtidos no presente estudo, indicaram baixos índices de infecção (Bush et al., 1997). 


\section{Conclusão}

Os exemplares analisados apresentaram parasitas associados ao tráfico de aves silvestres. A presença de espécies não registradas anteriormente (D. delirae e Heterakis $s p$.) sugere uma possível transmissão associada às más condições de captura e transporte do tráfico, que possibilitam o contato direto com outras espécies de animais silvestres. O presente trabalho amplia o conhecimento acerca da helmintofauna associada a $P$. dominicana, trazendo informações relevantes diante do interesse do tráfico de aves silvestres sobre esta espécie. Acredita-se que esses resultados possam auxiliar nas medidas de conservação de Passeriformes, como P. dominicana. Sugerimos fortemente que mais pesquisas sejam realizadas dentro do tema, para que em projetos futuros as aves tenham chance de tratamento em vida. Por fim, apelamos às instituições de pesquisa para que estudos como este, apoiando o trabalho do CETAS, sejam mais frequentes.

\section{Referências}

Aguirre, A. A. (2010). Parasitic diseases in wildlife and domestic animals: new trends of disease emergence. In P. C. Lefevre, J. Blancou, R. Chermette, \& G. Uilenberg (Eds.). Infectious and Parasitic Diseases of Livestock: General Considerations, Viral Diseases 1, 73-79. Lavoisier.

Alves, R. R. N., Lima, J. R. F., \& Araujo, H. F. P. (2012). The live bird trade in Brazil and its conservation implications: an overview. Bird Conservation International, 23(1), 53-65.

Anderson, R. C. (2000). Nematode parasites of vertebrates: their development and transmission (2a ed.). Wallingford: CABI.

Boll, A. S., Marques, S. M. T., \& Alievi, M. M. (2017). Parasitas em Passeriformes e Psittaciformes alojados em centro de triagem no Zoológico em Sapucaia do Sul, Rio Grande do Sul, Brasil. Revista Portuguesa de Ciências Veterinárias, 112(603-604), 28-34

Bush, A. O., Lafferty, K. D., Lotz, J. M., \& Shostak, A. W. (1997). Parasitology Meets Ecology on Its Own Terms: Margolis et al. Revisited. The Journal of Parasitology, 83(4), 575-583.

Carvalho, A. R., Lima, S. S., \& Daemon, E. (2006). Helmintofauna em cardeais, Paroaria dominicana (L.) (Passeriformes, Emberizidae): Taxonomia e ecologia de populações e de comunidades de parasitos. Revista Brasileira de Zoociências, 8(2), 207.

Carvalho, A. R., Daemon, E., \& Souza-Lima, S. (2007) Relação entre o peso do baço e infecção por helmintos em galo da campina Paroaria dominicana (Linnaeus, 1758) (Passeriformes, Emberizidae) do estado da Bahia, Brasil. Revista Brasileira de Zoociências, 9(2), $219-224$.

Costa, F. J. V, Ribeiro, R. E, De-Souza, C. A., \& Navarro, R. D. (2018). Espécies de aves traficadas no Brasil: uma meta-análise com ênfase nas espécies ameaçadas. Fronteiras: Journal of Social, Technological and Environmental Science, 7(2), 324-346.

Dalfovo, M. S., Lana, R. A., \& Silveira, A. (2008). Métodos quantitativos e qualitativos: um resgate teórico. Revista Interdisciplinar Científica Aplicada, 2(4), 01-13.

Destro, G. F. G., Pimentel, T. L., Sabaini, R. M., Borges, R. C., \& Barreto, R. (2012). Efforts to combat wild animals trafficking in Brazil. In G. A. Lameed (Ed.) Biodiversity enrichment in a diverse world (pp. 421-436). Londres: IntechOpen.

Karesh, W. B., Cook, R. A., Bennett, E. L., \& Newcomb, J. (2005). Wildlife Trade and Global Disease Emergence. Emerging Infectious Diseases, 11(7), 1000-1002.

Leape, J., \& Brito, M. C. W. (2013). Illegal trading threat species all over the world. https://www.wwf.org.br/informacoes/english/?33822/Illegal-tradingthreat-species-all-over-the-world

Mascarenhas, C. S. (2008). Helminto e artropodofauna de Paroaria coronata (Miller, 1776) (Passeriformes: Emberzidae). Dissertação de mestrado, Departamento de Microbiologia e Parasitologia, Instituto de Biologia, Universidade Federal de Pelotas, Pelotas, RS, Brasil. http://repositorio.ufpel.edu.br:8080/handle/123456789/2343

Morais, J., Souza, D., Gallas, M., Silveira, E., \& Périco, E. (2018). Diplotriaena delirae Pinto e Noronha, 1970 (Nematoda, Diplotriaenidae) in Pitangus sulphuratus (Linnaeus, 1766) (Passeriformes, Tyrannidae) from southern Brazil. Check List, 14(5), 823-826.

Pereira, A. S., Shitsuka, D. M., Parreira, F. J., \& Shitsuka R. (2018). Metodologia da pesquisa científica (1a ed.). [free e-book]. Santa Maria: Núcleo de Tecnologia Educacional da Universidade Federal de Santa Maria. https://repositorio.ufsm.br/handle/1/15824

Regueira, R. F. S., \& Bernard, E. (2012). Wildlife sinks: Quantifying the impact of illegal bird trade in street markets in Brazil. Biological Conservation, $149(1), 16-22$.

Renctas - Rede Nacional de Combate ao Tráfico de Animais Silvestres. (2001). $1^{o}$ Relatório nacional sobre o tráfico de fauna silvestre. http://www.renctas.org.br/wp-content/uploads/2014/02/REL_RENCTAS_pt_final.pdf

Rosa, A. L., Lima-Rezende, C. A., Rodrigues, F. P., \& Caparroz, R. (2019). Development and characterization of 19 polymorphic microsatellite loci from the Red-cowled Cardinal (Paroaria dominicana, Passeriformes, Aves) using next-generation sequencing. Molecular Biology Reports, $46,5531-5536$.

Sanches, T. C. (2008). Causas de morte em Passeriformes: comparação entre aves de vida livre residentes na Região Metropolitana de São Paulo e aves 
Research, Society and Development, v. 10, n. 12, e96101220164, 2021

(CC BY 4.0) | ISSN 2525-3409 | DOI: http://dx.doi.org/10.33448/rsd-v10i12.20164

oriundas do tráfico. Dissertação de mestrado, Faculdade de Medicina Veterinária e Zootecnia, Universidade de São Paulo, São Paulo, SP, Brasil. http://www.teses.usp.br/teses/disponiveis/10/10133/tde-02062008-102715/

Silveira, L. F. (2012). Mundo das aves: os cardeais brasileiros. Cães Cia, 399, 44-45.

Thompson, R. C. A, Lymbery, A. J., \& Smith, A. (2010). Parasites, emerging disease and wildlife conservation. International Journal for Parasitology, 40(10), 1163-1170.

Tomás, A. F. V. (2014). Rastreio parasitológico em aves selvagens de zonas periurbanas do litoral e interior de Portugal. Dissertação de mestrado, Departamento de Biologia Animal, Faculdade de Ciências, Universidade de Lisboa, Lisboa, Portugal. https://repositorio.ul.pt/handle/10451/15556

Vicente, J. J., Pinto, R. M., \& Noronha, D. (1983). Estudo das espécies brasileiras do gênero Diplotriaena Henry \& Ozoux, 1909 (Nematoda, Filarioidea). Memórias do Instituto Oswaldo Cruz, 78(2), 165-182.

Vicente, J. J., Rodrigues, H. O., Gomes, D. C., \& Pinto, R.M. (1995). Nematóides do Brasil. Parte IV: nematóides de aves. Revista Brasileira de Zoologia, 12(supl. 1), 1-273. 